\title{
How we treat Merkel cell carcinoma: within and beyond current guidelines
}

\author{
Song Y Park ${ }^{1}$ (D), Coley Doolittle-Amieva1 ${ }^{1}$ Yasman Moshiri ${ }^{1}$, Tomoko Akaike ${ }^{1}$ (D), Upendra

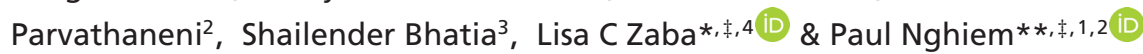 \\ ${ }^{1}$ Division of Dermatology, University of Washington at South Lake Union, Seattle, WA 98109, USA \\ ${ }^{2}$ Department of Radiation Oncology, University of Washington, Seattle, WA 98109, USA \\ ${ }^{3}$ Department of Medicine, University of Washington, Seattle, WA 98109, USA \\ ${ }^{4}$ Department of Dermatology, Stanford University, Palo Alto, CA 94304, USA \\ *Author for correspondence: Tel.: +1 650725 3292; Izaba@stanford.edu \\ **Author for correspondence: Tel.: +1 206221 2632; pnghiem@uw.edu \\ $¥$ Authors contributed equally
}

Merkel cell carcinoma (MCC) is an aggressive skin cancer associated with a high risk of local recurrence and distant metastasis. Optimal care of this potentially life-threatening cancer is critical but challenging because: physicians are often unfamiliar with its management due to rarity, and MCC management remains controversial, in part because it is rapidly evolving across multiple specialties. While guidelines offer a broad overview of management, they are often not sufficient when making decisions for individual patients. Herein, we present a literature review as well as practical approaches adopted at our institutions for staging, surveillance and therapy of MCC. Each of these areas are discussed in light of how they can be appropriately customized for prevalent but challenging situations. We also provide representative examples of MCC patient scenarios and how they were managed by a multidisciplinary team to identify suitable evidence-based, individualized treatment plans.

Lay abstract: Merkel cell carcinoma (MCC) is a skin cancer with a high risk of recurrence and distant spread. Optimal care of this cancer is important. However, management is challenging because it is rare and its treatment is continuously evolving across multiple specialties. While treatment guidelines offer a broad overview of management, they are often not detailed enough to provide appropriate patient-specific assistance. Herein, we present a review of recent studies and our suggestions relevant to MCC staging, surveillance and treatment options. Each of these areas are discussed in light of how they can be appropriately customized for challenging situations often encountered by practitioners. We also provide representative examples of MCC patient scenarios and how they were managed by a multidisciplinary team to identify evidence-based, individualized treatment plans.

First draft submitted: 12 October 2020; Accepted for publication: 8 December 2020; Published online: 29 January 2021

Keywords: customized treatment • imaging • immunotherapy • Merkel cell carcinoma • multidisciplinary management $\bullet$ radiotherapy $\bullet$ surgery $\bullet$ surveillance

Merkel cell carcinoma (MCC) is a rare but highly aggressive skin cancer. Approximately half of MCC patients present with nodal or distant metastatic disease at the time of diagnosis [1], and more than a third of patients experience recurrences despite initial treatment, with reported mortality rates of 33-46\% [2]. Risk factors for developing MCC include fair skin, ultraviolet (UV) exposure, immunosuppression and older age [3], and due to the aging population, the incidence is increasing with approximately 3000 cases/year in 2020 and approximately 3200 cases in 2025 in the USA [4].

In terms of its cause, MCC can be clearly delineated into two distinct developmental origins. In the USA, $80 \%$ of MCCs are caused by Merkel cell polyomavirus $(\mathrm{MCPyV})$ with a relatively low mutational rate, and the remaining $20 \%$ by extensive UV-mediated damage with a mutational rate nearly 100 -fold higher than virus-positive MCC [5].

Future Medicine 
MCC typically presents as a solitary, rapidly growing, nontender, red or violet nodule in an older or immunocompromised individual [3]. However, the clinical features are nonspecific, which often lead to confusion with a benign lesion such as a cyst [3].

Despite its increasing incidence and aggressive nature that require prompt intervention and diligent surveillance, optimal care for MCC is often challenging partly because many physicians are not familiar with MCC management due to its relatively low incidence. Furthermore, the field is continuously being updated across multiple subspecialty areas with controversies surrounding several management issues. Guidelines such as those published by the National Comprehensive Cancer Network (NCCN) represent consensus management of MCC. However, guidelines and review articles often leave gray areas in management because of the need to be appropriate for broad clinical scenarios but not necessarily for individual cases. Given the fact that guidelines must be quite general in nature, we believe that multidisciplinary care is essential to formulate a patient-specific management plan that reflects the state of the art for each medical specialty. In this article, in addition to referring to these established guidelines, we attempt to provide more context for decision-making based on the most current data and our experience.

\section{Role of a multidisciplinary team}

Optimal MCC care requires input from various specialties. As part of initial work-up and treatment of primary and/or recurrent disease, consultation with a specialized center would be beneficial. Close coordination between a specialized center and a local multidisciplinary team (ideally through a patient health information compliant videobased case conference) allows for informative collaboration between an academic center and a regional treatment center, facilitating treatment and surveillance close to the patient's home. For easy identification of regional or local expertise, we actively maintain an online list of over 70 domestic and international centers and their physician leaders that have a specialty interest in MCC care (https://merkelcell.org/centers).

The multidisciplinary care teams often include, but are not limited to dermatology, surgery, radiation oncology, radiology and interventional radiology, and medical oncology. Additional expertise or specialist services may be utilized if specific organ systems are infiltrated with MCC or if patients develop an immune-related adverse event from immune-checkpoint inhibitor treatment.

The collaborative decision-making process greatly affects treatment course in a majority of cases. In our experience, the preliminary treatment plan is significantly modified in more than half of cases after discussion in a multidisciplinary conference.

\section{Flow of MCC treatment}

Our general approach for treating newly diagnosed MCC is outlined in the flow chart shown in Figure 1. After initial diagnosis, obtaining a baseline MCPyV oncoprotein antibody titer should be considered for prognostic significance and surveillance (please see 'MCPyV oncoprotein antibodies as a tumor marker' section below). Multiple studies suggest that obtaining a baseline imaging study before MCC excision and sentinel lymph node biopsy (SLNB) is indicated in most cases (please see 'Significance of baseline imaging prior to surgery' section below). Staging workup and treatment are different depending on whether patients present with clinically positive or negative lymph nodes. Excision of the primary MCC site is generally indicated if the lesion is surgically resectable. Guidelines for appropriate margins are controversial, especially in the context of possible adjuvant radiation therapy (RT) after surgery (please see 'Wide local excision versus narrow excision' section). For large primary MCCs, if surgical resection is not feasible, definitive RT can be considered as an alternative option. In such cases, the in-field disease control rate for definitive RT (60-66 Gy) is about 70-80\% [6]. For patients with no palpable or radiologically detectable lymph node involvement, we recommend SLNB for most patients (please see 'Sentinel lymph node biopsy' section below). Treatment of the lymph node basin is indicated in high-risk patients. Tumor-involved lymph nodes can be treated with surgery and/or RT (please see 'Treatment for draining lymph node basin or involved lymph nodes' section).

PD-1/PD-L1 inhibitors are the treatment of choice for metastatic or unresectable MCC (please see 'Treatment strategy for locally unresectable and metastatic disease' section).

\section{MCPyV oncoprotein antibodies as a tumor marker}

The majority ( $\sim 80 \%)$ of MCC tumors in the USA are caused by MCPyV [5]. Antibodies that recognize MCPyV oncoproteins are found in approximately $50 \%$ of MCC patients, and patients who produce antibodies have a better prognosis compared with antibody-negative patients $[7,8]$. In addition, in patients who have baseline positive 


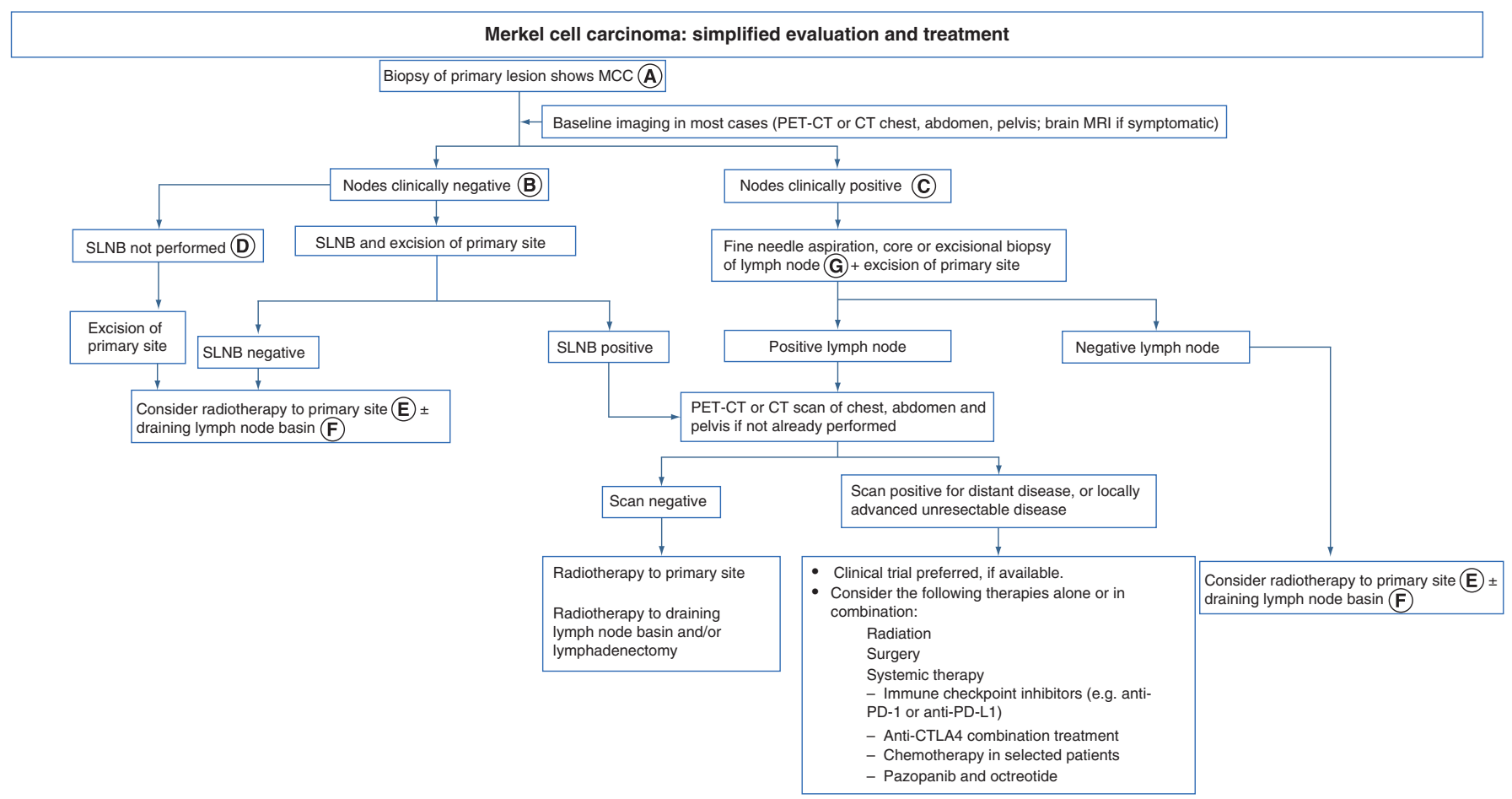

Figure 1. Merkel cell carcinoma: simplified evaluation and treatment. (A) Consider baseline Merkel cell polyomavirus oncoprotein antibody test for prognostic significance and to track disease. (B) No pathologically enlarged nodes on physical examination and by imaging study. (C) Pathologically enlarged nodes on physical examination or by imaging study. (D) Select patients may not undergo SLNB (e.g., patients who cannot tolerate anesthesia for SLNB or who prefer no SLNB due to their medical condition). In those patients, treatment for draining lymph nodes can be considered based on the local recurrence risk. (E) Radiotherapy to the primary site is indicated in most patients; the exception is for low-risk disease (e.g., primary $\leq 1 \mathrm{~cm}$, lesion on the extremities or trunk, no lymphovascular invasion, widely negative surgical margin and no chronic T-cell immunosuppression). (F) Consider radiation therapy to the nodal basin in high-risk patients or if SLNB dye did not migrate as expected. (G) Consider excisional biopsy primarily or after negative needle/core biopsy to exclude false-negative biopsy result.

SLNB: Sentinel lymph node biopsy; PET: Positron emission tomography.

$\mathrm{MCPyV}$ antibody titers, changes in titers over time reflect changes in MCC disease burden, making MCPyV antibody surveillance a useful serum tumor marker [7,9].

Ideally, a baseline test should be performed within 3 months of treatment because the titer decreases after successful treatment of MCC. If patients do not produce antibodies at baseline, they have approximately $42 \%$ increased risk of recurrence [7]. For such seronegative patients, an immunohistochemistry test for MCPyV large T-antigen expression within tumor cells can be considered if indicated (e.g., for additional prognostic information, evaluation of clinical trial eligibility, etc.) [10]. Hence, patients who do not produce MCPyV oncoprotein antibodies and/or whose tumor is negative for MCPyV large T-antigen immunohistochemistry stain will benefit from regular imaging surveillance to detect early disease recurrence.

If patients produce antibodies, it can be concluded that their MCC was caused by MCPyV because control subjects do not produce these antibodies. MCPyV antibody testing can optimize surveillance in conjunction with imaging studies, because: an increasing titer prompts imaging studies to confirm and localize recurrence in a timely manner; a decreasing titer indicates that there is no recurrence or that tumor burden is reduced significantly.

Recent data from our cohort suggest that the test is highly sensitive and specific. An analysis of 254 patients indicates that two consecutive increasing titers of more than $20 \%$ have a positive predictive value for disease recurrence of approximately $99 \%$ (95\% CI: 94-100\%). 90\% of recurrences were detected within 45 days of the first increased titer. In contrast, a titer that decreases by more than $20 \%$ has a $99 \%$ negative predictive value $(95 \%$ CI: 96-100\%) for the presense of clinically detectable disease [9].

For patients who produce $\mathrm{MCPyV}$ antibodies, we repeat the test every 3 months to assess disease status. A detailed algorithm for MCPyV oncoprotein antibody testing is shown in Figure 2. 
Suggested algorithm to interpret MCPyV oncoprotein antibody test

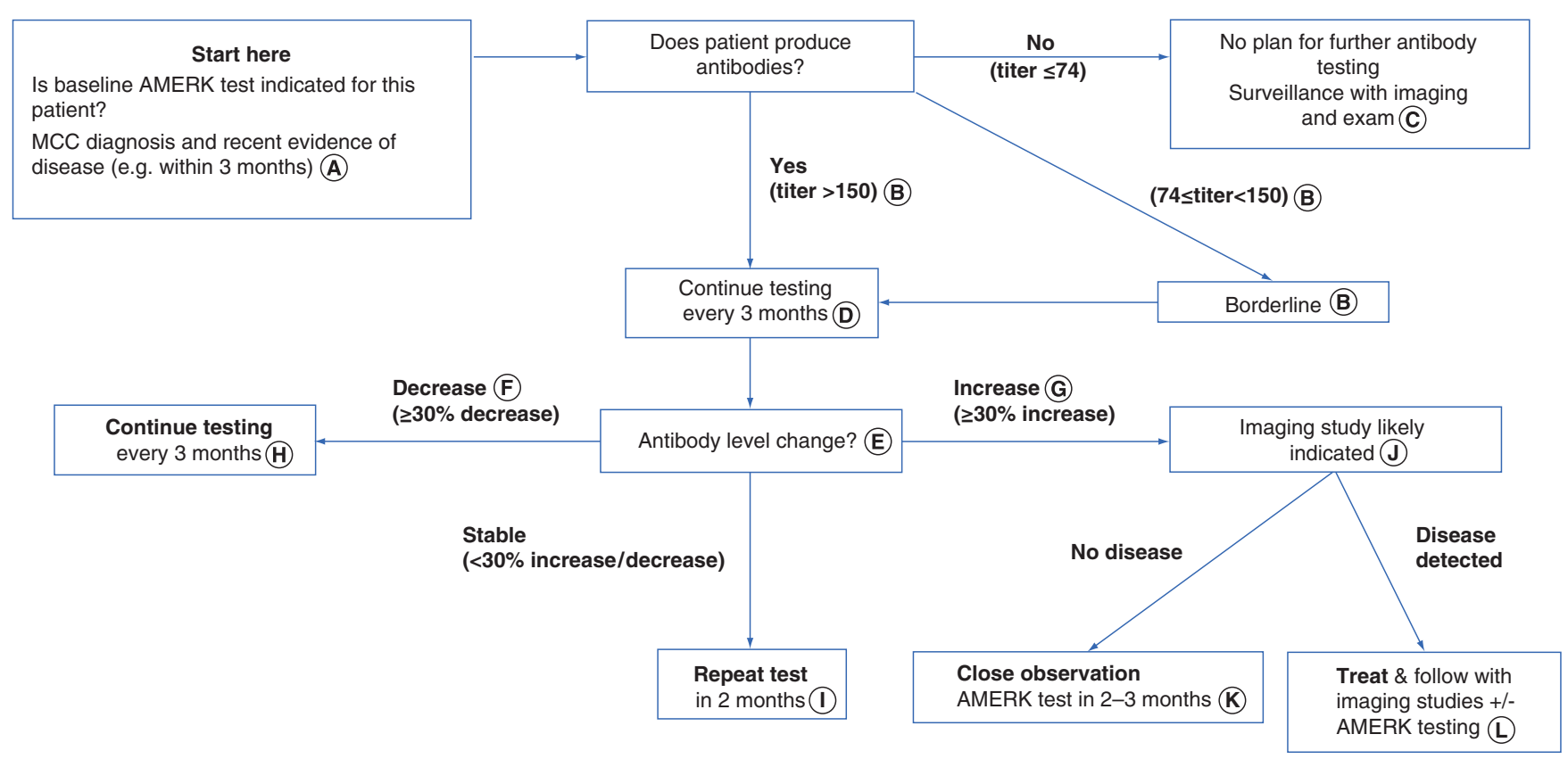

Figure 2. Suggested algorithm to interpret Merkel cell polyomavirus oncoprotein antibody test (AMERK test). (A) For seropositive patients, the AMERK titer decreases significantly within 3 months after successful treatment. A late baseline test that comes back negative $(<74)$ may indicate that: the patient produced antibodies initially, but the antibody levels decreased to undetectable range after the tumor was removed, or the patient does not produce antibodies to MCPyV oncoprotein. In contrast, if the patient produces antibodies at any time after diagnosis, this test is positive and could be used for future surveillance. (B) AMERK titer between 74 and 150 is considered to be 'borderline'. In our practice, this typically represents seropositivity, particularly if subsequent titer changes out of the borderline range. If titer still stays within borderline range in subsequent draws, AMERK titer for the patient is not reliable. (C) If the baseline test was done after more than 3 months from the initial diagnosis, or the size of MCC tumor at the time of the baseline test was extremely small (e.g., $<5 \mathrm{~mm}$ ), we consider repeating the AMERK test if the patient recurs in the future. (D) The AMERK test is most reliable for surveillance on patients who have not experienced a recurrence, or for detecting their first recurrence. After multiple recurrences and/or immunotherapy (systemic or local), the titer change of the AMERK testing is less reflective of disease burden. (E) Individual baseline titer could vary from less than 74 to more than 115,000 . While this flowchart is designed to guide clinicians to interpret a test result at a single timepoint compared with the previous one, we find that consistent trends over time better correspond to disease status in the clinical setting. For instance, if the patient's AMERK titer is slowly trending upward, we suggest interpreting the result as an 'increase' of titer and considering imaging studies. (F) In general, for the first 12 months following diagnosis, we recommend the patients have regular scans per the initial recurrence risk as determined by their disease stage and other factors

(https://merkelcell.org/prognosis/recur/). In our practice, if the AMERK titer decreases by more than $70 \%$ from baseline, shows decreasing or stable trend for several consecutive draws, and the patient is more than 12 months out from their original diagnosis, we consider that AMERK can safely substitute for scans for surveillance. (G) Most patients that have two antibody tests with increased titers (e.g. $\geq 30 \%$ change) will eventually have MCC recurrence [9]. (H) Recurrence risk depends on stage at diagnosis and other patient characteristics. For most patients, it decreases dramatically by 2 years after diagnosis (https://merkelcell.org/prognosis/recur/). We generally recommend discontinuing or decreasing frequency of imaging and/or AMERK studies when a patient's individual residual risk of recurrence is below $2 \%$, or by 5-6 years after initial diagnosis. (I) To establish if either increasing or decreasing trend is outside of the margin of error. (J) PET/CT or CT with contrast could be considered. Some studies and our experience indicate that PET/CT has higher sensitivity. If imaging studies fail to locate disease repeatedly in the setting of rising antibodies, consider alternative scan modalities (e.g., MRI) or investigate other body parts (e.g., brain, extremities that were not included in the previous scan). (K) Concurrent imaging test may be indicated. If no clinically evident disease is detected in the setting of AMERK titer increase, we suggest considering more sensitive scan modalities (e.g., PET/CT) or evaluating anatomical site that has not been evaluated by imaging (e.g., extremities, brain). (L) The additive benefit of the AMERK test when patients are receiving routine scans is unknown.

CT: Computed tomography; MCC: Merkel cell carcinoma; MCPyV: Merkel cell polyomavirus; PET: Positron emission tomography.

Based on the clinical utility of this test, NCCN guidelines for MCC suggest that clinicians consider determining $\mathrm{MCPyV}$ oncoprotein antibody titer as part of initial work-up of MCC and for ongoing surveillance in seropositive patients [11]. The test is clinically available in the USA and Europe (updated logistical information is available through the institutions running the tests $[12,13])$. 


\section{Significance of baseline imaging prior to surgery}

Current recommendations including the NCCN guidelines suggest that baseline cross-sectional imaging studies, for example, computed tomography (CT) or positron emission tomography (PET)/CT, to be performed as 'clinically indicated', such as when metastatic disease is suspected by physical exam, or lymph node biopsy confirms disease involvement [11]. This recommendation was adopted based on data from malignant melanoma, which suggests that only a small fraction $(<1 \%)$ of imaging studies reveal metastatic disease for patients with clinically localized melanoma [14,15].

In MCC, however, several studies indicate that baseline imaging regardless of physical exam findings including clinical nodal involvement often impacts management [16-19]. In a recent study of 584 patients, baseline imaging detected occult metastatic disease in $13 \%$ of cases presenting without suspicious physical exam findings $(\mathrm{n}=492)$, which thereby resulted in upstaging. In addition, among patients who did have clinical nodal involvement, $11 \%$ of 92 patients had upstaging to distant metastatic disease [20]. Given that one in eight patients has significant upstaging of MCC, regardless of clinical nodal status, we perform baseline imaging prior to surgery or SLNB in virtually all patients (Figure 1).

In terms of the scan modalities, PET with fluorodeoxyglucose (FDG), combined CT and/or MRI, has been reported to be more sensitive compared with CT alone [16-19]. In the study of 584 patients mentioned above, 17\% of patients who underwent PET-CT imaging were upstaged, as compared with $7 \%$ of those who received CT only ( $p=0.0006)$ [20]. Surveillance scanning will be discussed in the section 'Surveillance.'

\section{Wide local excision versus narrow excision}

Local treatment of MCC often includes wide excision of the primary site, which can lead to significant morbidity and delayed wound healing.

Despite many retrospective series being published [21-24], the effect of surgical margin size on local recurrence or survival remains controversial. Adjuvant RT is well known to diminish the risk of local recurrence of MCC [25]. Despite this, most prior studies have not separately analyzed outcomes of patients based on whether or not adjuvant RT was given.

A few recent studies did such analysis and found that adjuvant RT is indeed powerful at controlling narrow or even positive surgical margins [26,27]. In a study of 179 Canadian patients, among these who had a narrow excision $(<1 \mathrm{~cm})$, adjuvant RT was associated with a lower local recurrence rate $(25 \mathrm{vs} 5 \%, \mathrm{p}=0.03)$. For patients who had more than $1 \mathrm{~cm}$ margins, local recurrence was $7 \%$ regardless of whether the patient received adjuvant RT or not. In a separate study of 188 patients who presented without clinical nodal involvement, surgical margins of more than $1 \mathrm{~cm}$ were associated with a lower local recurrence rate among patients who underwent surgery alone. However, for patients who received adjuvant RT, local control was excellent (1\% local recurrence) regardless of surgical margin size even for patients who had microscopically positive margins [27].

As such, we believe that surgical margins should be considered in the context of possible adjuvant RT. Patients with very low-risk disease who have all of the following six favorable prognostic factors (primary tumor $\leq 1 \mathrm{~cm}$, no chronic T-cell immunosuppression, lesion not on head/neck, no lymphovascular invasion, confidently negative surgical margins and negative SLNB) do not benefit from adjuvant RT. However, some of the criteria for decision on adjuvant RT are unknown at the time of surgical excision (e.g., SLNB status, histologic margins following re-excision). Hence, we propose a practical algorithm that guides surgical margin decision-making for primary tumor excision of local MCC (no clinical nodal involvement or in-transit disease) (Figure 3).

\section{Sentinel lymph node biopsy}

For patients with no palpable or radiologically detectable lymph node involvement ('Nodes clinically negative' in the flowchart), we generally recommend SLNB for all patients who can undergo general anesthesia, regardless of tumor size or location, because determining tumor involvement of lymph nodes informs staging and prognosis and guides decisions regarding regional adjuvant RT or nodal dissection.

Unlike melanoma in which SLNB is indicated only among higher-risk tumors, SLNB positivity for MCC at initial diagnosis is estimated to be $20-50 \%$ depending on the tumor size. Patients with primary lesions on the trunk, tumor-infiltrating lymphocytes or lymphovascular invasion are more likely to have positive sentinel lymph nodes [28]. It is important to note that even a small tumor $\leq 0.5 \mathrm{~cm}$ can have positive SLN in approximately $15 \%$ of cases [29]. However, SLNB for head and neck tumors has a higher false-negative or technically unsuccessful result due to the complex lymphatic drainage in the area [30]. 


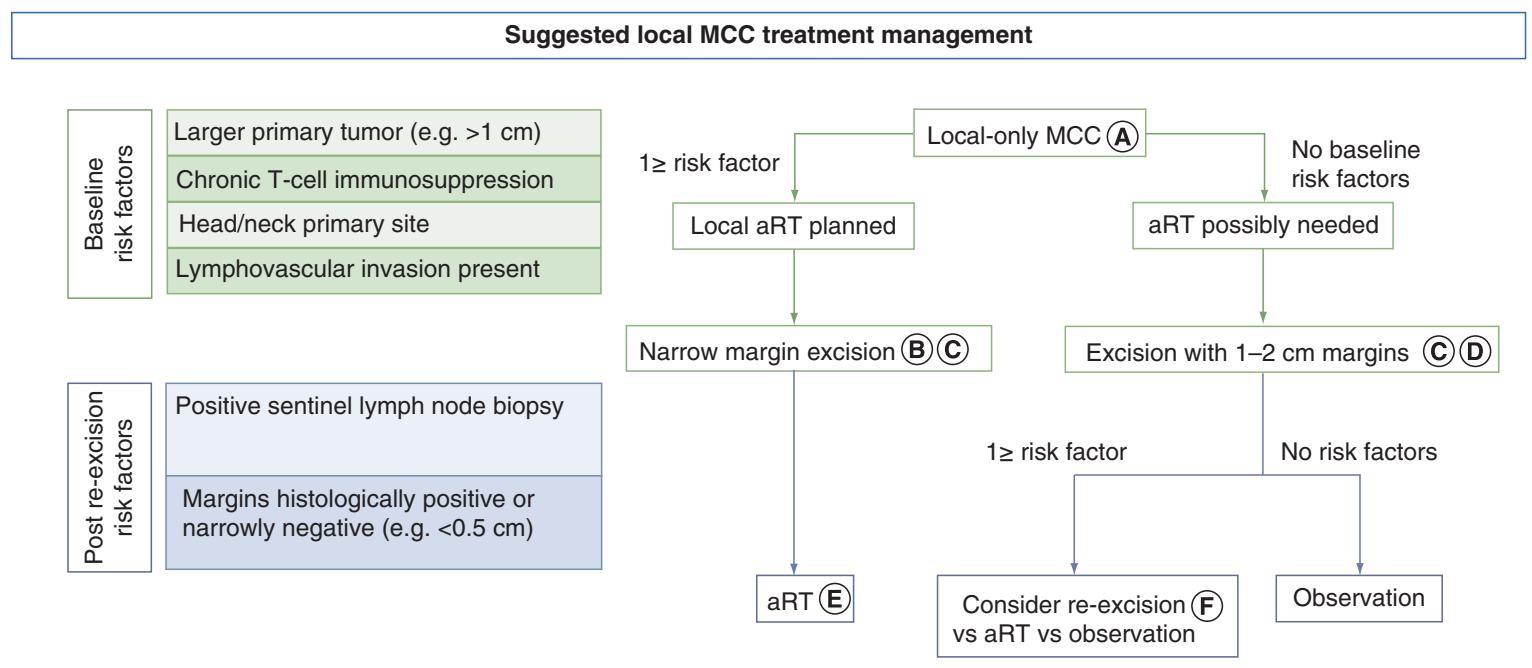

Figure 3. Suggested local Merkel cell carcinoma treatment management. Flowchart of local treatment of surgery and radiation that integrates risk factors that are associated with local recurrence. Certain risk factors are available at time of diagnosis (baseline), while others are only available after surgical excision (post re-excision). (A) Criteria for 'local only MCC' were clinically node-negative, no in-transit disease, and imaging negative for distant disease. (B) Sentinel lymph node biopsy typically performed at this time. (C) Narrow excision margins minimize morbidity and if aRT is performed microscopically positive margins are acceptable. (D) Goal should be primary tissue closure (i.e., without flap or graft) allowing aRT initiation within 3-4 weeks. (E) If sentinel lymph node biopsy positive, nodal aRT would typically be given, in addition to primary site aRT. (F) Decision on re-excision based on clinical setting (narrow path margins, e.g., $<0.5 \mathrm{~cm}$ ) and patient preference, re-excision versus aRT versus observation. aRT: Adjuvant radiation treatment; MCC: Merkel cell carcinoma.

Adapted with permission from [27].

Radiotracer for lymphatic mapping should be injected ideally before excision of the primary MCC site because surgery can change lymphatic drainage patterns. Typically, SLNB is performed during the same surgery visit as wide local excision (WLE).

\section{Treatment for draining lymph node basin or involved lymph nodes}

For patients who have a negative SLNB, elective RT to the draining lymph nodes can be omitted in most cases. For patients who elect not to have SLNB or if it cannot be performed due to co-morbidities that increase risk of general anesthesia, WLE with or without adjuvant RT is similarly considered depending on individual known risk factors for local or regional recurrence. For patients with clinical nodal involvement, pathologic confirmation of lymph node involvement is recommended.

Excision and/or adjuvant RT to the primary site are indicated for disease with microscopic (SLNB positive) or macroscopic (clinically detectable) lymph nodes involvement. For microscopic disease involvement of lymph nodes, definitive RT or completion lymphadenectomy to the lymph node basin is recommended. We do not recommend performing both nodal RT and completion nodal dissection for microscopic disease because the combination does not improve local control and significantly increases morbidity [31]. In patients with macroscopic nodal disease, RT and/or lymphadenectomy is indicated to the nodal basin. Excision of the clinically abnormal node followed by RT is one reasonable approach. Completion lymphadenectomy is also a reasonable approach depending on the clinical scenario [11]. Definitive RT without lymph node removal is also an appropriate option [31].

\section{Potential role for hypofractionated RT}

MCC has long been known to be highly radiosensitive [32]. Conventional RT is typically delivered with a total dose of 50-66 Gy over 4-6 weeks. The morbidity of this radiation dose is often significant including acute skin reactions, mucositis, chronic skin and soft tissue fibrosis, or long-term functional damage (e.g., severe dry eye, diminished saliva production). Daily treatments over several weeks can also be a significant logistical challenge [33].

Hypofractionated RT such as single-fraction RT (SFRT) with 8 Gy has been used in the palliative setting for MCC, with local response rate of $94 \%$. SFRT also has a superior safety profile [34,35] with minimal or no morbidity in the vast majority of treated cases. 
Based on the high efficacy, favorable side effect profile and logistical convenience, SFRT in the adjuvant setting has been investigated in a subset of patients, such as those who cannot tolerate conventional radiation treatment, or who wish to avoid radiation side effects. In a study of 12 patients with localized MCC of the head and neck who elected SFRT for postoperative adjuvant RT, at a median follow-up of 642 days (range: 263-2909), no in-field locoregional recurrences were reported. A single out-of-field regional recurrence was observed, which was successfully salvaged. No radiation-associated toxicities greater than grade 1 (Common Terminology Criteria for Adverse Events version 5) were observed [33].

SFRT could be considered in patients with relatively low-risk of local recurrence, especially if the tumor is located in an area where higher RT morbidity is expected, such as near the eyelids or nose, or if existing comorbidities restrict full-dose adjuvant RT. An additional attractive feature of using SFRT is that salvage options are not compromised in case of an in-field recurrence.

\section{Treatment strategy for locally unresectable \& metastatic disease}

PD-1/PD-L1 inhibitors should be considered the first-line treatment of choice for patients with advanced or metastatic MCC who do not have contraindications to checkpoint-inhibitor therapy, which is supported by multiple clinical trials demonstrating durable response from these agents [11]. The response rate to PD-1/PDL1 inhibitors is approximately $50-70 \%$ for first line, and approximately $30 \%$ for the second or higher-lines of therapy [36-40]. In contrast to conventional chemotherapy for which less than $5 \%$ of responders continue to benefit at 2 years after starting therapy [41], among patients who initially respond to PD-1/PD-L1 inhibitors as a first- or later-line treatment, response durability at 2 years is estimated to be approximately $70-80 \%$ [36-40].

Based on those data, the US FDA has approved avelumab and pembrolizumab for treatment of patients with locally advanced or metastatic MCC, irrespective of prior therapy.

Inspired by success of PD-1/PD-L1 inhibitors in advanced MCC, use of immune checkpoint inhibitors as neoadjuvant and adjuvant treatments is currently being investigated in clinical trials. In the neoadjuvant setting, a recent study evaluated 39 MCC patients with higher risk local or regional disease. They received two doses of nivolumab followed by definitive surgical management. Around 50\% patients demonstrated radiographic or pathologic complete response (CR). The recurrence-free survival significantly correlated with disease response at the time of surgery. While additional studies are required, neoadjuvant therapy in MCC could potentially decrease the extent of local treatment in select patients [42]. In addition, adjuvant PD-1/PD-L1 inhibitor therapy is being investigated in multiple trials in the USA, Europe and Australia (ClinicalTrials.gov: NCT02196961, NCT03271372, NCT03712605, NCT04291885).

Despite the encouraging success of PD-1/PD-L1 inhibitors, approximately $50 \%$ of patients have either initial nonresponsive disease (primary or intrinsic resistance) or develop progression after initial benefit (secondary or acquired resistance). Additionally, a proportion of patients has absolute contraindications to immune checkpoint inhibitor (ICI) therapy including pre-existing severe autoimmune disease or ongoing immunosuppression due to solid organ transplant. Current treatment options are limited for patients who are not eligible for PD-1/PD-L1 inhibitors, or whose disease has progressed while on the drugs. Some treatment options are summarized as follows:

- Clinical trials: patients should be encouraged to participate in clinical trials whenever feasible. There are several clinical trials ongoing for PD-1 refractory MCC (Table 1);

- Chemotherapy: while the response rate to first-line chemotherapy is approximately 50-60\% [41,43], responses are short-lived with a median duration of 90 days from the start of therapy. In our practice, we prioritize clinical trials or other options first and typically reserve chemotherapy for palliation of rapidly growing disease or as bridge therapy to a subsequent treatment;

- SFRT or hypofractionated RT: for oligometastatic disease or symptomatic tumor masses, hypofractionated RT can be considered as an option, especially if an effective systemic therapy is not available or to debulk the tumor prior to starting a subsequent systemic therapy. In a retrospective analysis of MCC treated with SFRT, the objective response rate in irradiated tumors was 94\% [34]. Hypofractionated RT could also be used in conjunction with a PD-1/PD-L1 inhibitor to control refractory disease. In-field and abscopal responses have been reported in patients with metastatic MCC progressing on PD-1 checkpoint blockade [44];

- Ipilimumab: ipilimumab is an antiCTLA-4 agent, which could potentially synergize with a PD-1/PD-L1 inhibitor in MCC. In a retrospective case series of 13 patients whose MCC progressed through PD-1/PD- 
L1 inhibitors, who were treated with ipilimumab monotherapy or in combination with anti-PD-1, objective responses were reported in four patients [45];

- Intralesional treatment: MCC tumors that are located superficially (e.g., skin or superficial lymph nodes, which can be found by ultrasound) are ideal candidates for intralesional injection treatment. In a trial of G100, a Toll-like receptor- 4 agonist, the drug induced antitumor immune responses that were associated with partial responses (PRs) lasting 33+ months in two out of seven patients with metastatic MCC [46]. Intratumoral delivery of IL-12 with electroporation was investigated in a clinical trial. Among 12 patients with metastatic MCC, three patients (25\%) responded, two of whom had durable clinical benefit (16 and 55+ months, respectively) [47]. Among commercially available agents, talimogene laherparepvec (T-VEC) has been reported to be successful in several case reports including PD-1/PD-L1 inhibitor-refractory MCC [48-52]. Several clinical trials are currently available including T-VEC, Toll-like receptor-9 agonist or vaccines (NCT02819843, NCT03684785);

- Pazopanib: pazopanib is a tyrosine kinase inhibitor that demonstrated responses in several patients with metastatic MCC [53,54]. A trial of pazopanib in metastatic MCC reported clinical benefit in nine of 16 patients (three PRs and six with stable disease). Median progression-free survival time was 3.2 months (95\% CI: 1.4, 5.2) [54]. Subsequently, an observational case series reported clinical benefit in five out of 24 patients (one CR, three stable disease for 5 months to 3.5 years) who were treated with pazopanib for their metastatic MCC [53];

- Somatostatin analogs: a subset of MCC tumors expresses somatostatin receptors and can benefit from somatostatin analogue therapy [55]. Like other neuroendocrine tumors, peptide receptor radionuclide therapy with lutetium-177 isotope has been successfully used [56,57]. Octreotide and lanreotide have also been utilized for MCC. In a study of seven patients with metastatic MCC, three patients experienced progression-free survival of more than 120 days (median 237 days) [55], which is encouraging compared with the median benefit of approximately 90 days with conventional chemotherapy;

- Switching PD-1 and PD-L1: although slightly different mechanisms of action may suggest that switching therapy in between PD-1 and PD-L1 agents might be beneficial, there are no data to suggest that this is effective in clinical practice. In our practice, we do not typically switch between PD-1 pathway-blocking agents (without other significant changes in combination therapy) unless insurance or logistical issues arise.

For patients who benefit from PD-1/PD-L1 inhibitor, a paucity of data exists in terms of defining the appropriate duration of ICI after achieving CR or PR. Most clinical trials treat patients for 1-2 years. In our preliminary analysis of 59 patients who received PD-1/PD-L1 inhibitors with CR or PR and then discontinued therapy, 33\% of patients recurred after discontinuation $(n=20)$. One potential strategy to avoid disease recurrence following discontinuation of incompletely treated MCC would be to continue administration of PD-1/PD-L1 inhibitors for a longer period but at markedly lower frequency (e.g., every 3 months). Such a concept needs to be explored in detail.

\section{Immunosuppressed patients}

Hematologic malignancy, autoimmune disease with long-term immunosuppressive therapy, HIV/AIDS and solid organ transplantation are forms of T-cell immune-suppression and are present in approximately $10 \%$ of MCC cases [3,58]. MCC incidence varies depending upon the method of immunosuppression, and is approximately 40fold increased in chronic lymphocytic leukemia (CLL) patients [3], approximately 13-fold increased in HIV/AIDS patients [59] and approximately 25 -fold increased in solid organ transplant recipients [60]. Furthermore, immunosuppressed (IS) patients have significantly worse survival compared with immunocompetent patients [33,61].

Several treatment strategies can be considered for IS patients. For patients who are taking an immunosuppressant for their underlying condition (e.g., autoimmune disease, organ transplantation), we recommend modification or removal of immunosuppressive drugs whenever possible. Spontaneous regression of metastatic MCC by removal of iatrogenic-immunosuppression has been reported [62]. However, limited data exist to guide optimal selection of immunosuppressive drugs that balance antitumor immunity and control of the underlying condition [63]. From the malignancy risk standpoint, glucocorticoids, TNF- $\alpha$ inhibitors or methotrexate have been believed to be relatively safe in this setting [63-65]. Drugs with T-cell inhibitory mechanism (e.g., cyclosporine) or DNA synthesis inhibitors (e.g., azathioprine, cyclophosphamide) are thought to confer higher risk [64]. However, the level of evidence is low and heterogeneous depending on the type of immunosuppressive condition, cancer type, etc. Comanagement of immunosuppression by careful multidisciplinary collaboration is critical for optimally adjusting immune suppression. 
Table 1. Key clinical trials for advanced Merkel cell carcinoma treatment.

\begin{tabular}{|c|c|c|c|}
\hline Rank & NCT number & Title & Note \\
\hline \multicolumn{4}{|c|}{ Adjuvant therapy } \\
\hline 1 & NCT03271372 & Adjuvant avelumab in MCC (ADAM) & $\begin{array}{l}\text { Phase III, placebo-controlled, in the USA in high-risk MCC (stage } \\
\text { III only); duration of therapy: } 2 \text { years }\end{array}$ \\
\hline 2 & NCT02196961 & $\begin{array}{l}\text { Adjuvant therapy of completely resected MCC with immune } \\
\text { checkpoint blocking antibodies vs observation (ADMEC-O) }\end{array}$ & $\begin{array}{l}\text { Phase II trial in Germany using nivolumab in resected high-risk } \\
\text { MCC (all stages); duration of therapy: } 1 \text { year }\end{array}$ \\
\hline 3 & NCT03712605 & $\begin{array}{l}\text { Pembrolizumab compared with standard of care observation in } \\
\text { treating patients with completely resected stage I-III MCC } \\
\text { (STAMP) }\end{array}$ & $\begin{array}{l}\text { Phase III, in the USA in resected MCC (all stages); duration of } \\
\text { therapy: } 1 \text { year }\end{array}$ \\
\hline 4 & NCT04291885 & $\begin{array}{l}\text { Immunotherapy adjuvant trial in patients with stage I-III MCC } \\
\text { (I-MAT) }\end{array}$ & $\begin{array}{l}\text { Phase II, placebo-controlled, in Australia using avelumab in } \\
\text { high-risk MCC (stages I-III); duration of therapy: } 6 \text { months }\end{array}$ \\
\hline \multicolumn{4}{|c|}{ Metastatic MCC: systemic therapy } \\
\hline 1 & NCT03747484 & $\begin{array}{l}\text { Gene-modified immune cells (FH-MCVA2TCR) in treating } \\
\text { patients with metastatic or unresectable MCC (ATTAC-MCC) }\end{array}$ & Autologous T-cells with engineered TCR against MCPyV; $n=16$ \\
\hline 2 & NCT03599713 & A study of retifanlimab in metastatic Merkel cell carcinoma & Retifanlimab: anti-PD-1; $\mathrm{n}=90$ \\
\hline 3 & NCT03071406 & $\begin{array}{l}\text { Randomized Study of nivolumab }+ \text { ipilimumab } \pm R T \text { for } \\
\text { metastatic MCC }\end{array}$ & $\begin{array}{l}\text { Single-institution; PD-1 naive and PD-1 refractory cohorts; } \\
n=50\end{array}$ \\
\hline 4 & NCT03787602 & $\begin{array}{l}\text { KRT-232 for the treatment of patients with ( } \mathrm{p} 53 \mathrm{WT} \text { ) MCC who } \\
\text { have failed anti-PD-1/PD-L1 immunotherapy }\end{array}$ & KRT-232: MDM2-p53 inhibitor; $n=27$ \\
\hline 5 & NCT03853317 & $\begin{array}{l}\text { QUILT-3.063: a study of N-803, haNK and avelumab in patients } \\
\text { with MCC that has progressed after checkpoint therapy }\end{array}$ & $\begin{array}{l}\text { Phase II; } n=43 ; n-803 \text { : IL-15 super-agonist, haNK: off-the-shelf } \\
\text { CD16-targeted natural killer cells }\end{array}$ \\
\hline 6 & NCT04393753 & $\begin{array}{l}\text { Domatinostat in combination with avelumab in patients with } \\
\text { advanced MCC progressing on anti-PD-(L)1 }\end{array}$ & $\begin{array}{l}\text { Domatinostat: histone deacetylase inhibitor; } n=40 \text {; Europe and } \\
\text { US sites }\end{array}$ \\
\hline 7 & NCT04261855 & Targeted therapy and avelumab in MCC & $\begin{array}{l}\text { Avelumab + Lu-177DOTATATE in MCC expressing SSTR; } \\
\text { Australian sites }\end{array}$ \\
\hline \multicolumn{4}{|c|}{ Metastatic MCC: intralesional immunotherapy } \\
\hline 1 & NCT02819843 & A study of T-VEC with or without RT for MCC and melanoma & T-VEC: oncolytic virus; $\mathrm{n}=34$ \\
\hline 2 & NCT02978625 & $\begin{array}{l}\text { T-VEC and nivolumab in treating patients with refractory } \\
\text { nonmelanoma skin cancers }\end{array}$ & T-VEC: oncolytic virus; $n=68$ \\
\hline 3 & NCT04160065 & Immunotherapy with IFx-Hu2.0 vaccine for advanced MCC & $\begin{array}{l}\text { IFx-Hu2.0: pDNA-encoding autologous cancer cell vaccine; } \\
n=20\end{array}$ \\
\hline 4 & NCT03684785 & $\begin{array}{l}\text { Intratumoral cavrotolimod combined with pembrolizumab or } \\
\text { cemiplimab in patients with advanced solid tumors }\end{array}$ & Cavrotolimod: Toll-like receptor 9 agonist; $\mathrm{n}=180$ \\
\hline \multicolumn{4}{|c|}{ Miscellaneous } \\
\hline 1 & NCT03816332 & $\begin{array}{l}\text { Tacrolimus, nivolumab and ipilimumab in treating kidney } \\
\text { transplant recipients with selected unresectable or metastatic } \\
\text { cancers }\end{array}$ & Kidney transplant patient \\
\hline 2 & NCT03816345 & $\begin{array}{l}\text { Nivolumab in treating patients with autoimmune disorders or } \\
\text { advanced, metastatic or unresectable cancer }\end{array}$ & Autoimmune disease patient \\
\hline
\end{tabular}

For IS patients who require systemic treatment, PD-1/PD-L1 blockade is considered to be first-line therapy in patients without a contraindication. However, data for response rate in this population are sparse. In one study of 50 IS patients treated with PD-1/PD-L1 inhibitors for metastatic MCC [66], there was a 56\% response rate, which is similar to that of immunocompetent patients [40]; however, the response rate in CLL patients was only $18 \%$.

Pre-existing autoimmunity in a patient with advanced MCC presents a particularly difficult challenge given that immune checkpoint inhibition may worsen the underlying autoimmune disease. In a study of 52 melanoma patients with pre-existing autoimmune disease, $38 \%$ patients had a flare of disease after ICI treatment. Moreover, if continued immunosuppression is required during the initiation phase of the immunotherapy, it may decrease the response rate [67]. Despite those limitations, we consider ICI for patients with autoimmune disease in the setting of metastatic MCC because of its durability of response. An ongoing prospective trial to evaluate the safety and efficacy of immunotherapy in autoimmune disease patients is currently underway (NCT03816345).

\section{Surveillance}

While MCC is known to have a high rate of recurrence in general [2], individual risk of recurrence differs by stage ( $20 \%$ for stage I disease to $75 \%$ for stage III disease) and other patient-specific risk factors such as age, gender, 
immunosuppression, $\mathrm{MCPyV}$ status or tumor location [68]. In addition, the risk of recurrence decreases over several years with increasing time since initial diagnosis and treatment. A majority $(\sim 80 \%)$ of recurrences occur within 2 years of the initial diagnosis [69]. However, the rate of decrease in risk is significantly different based on the stage and patient-specific risk factors.

As such, it is important to discuss an individual patient's prognosis and recurrence risk at the time of initial management. Follow-up should also be individualized according to current recurrence risk, potential therapeutic options and patient preference. An individualized, web-based risk calculator, which integrates several relevant factors mentioned above, can assist in determining a patient's risk of recurrence and the appropriate frequency of surveillance studies (https://merkelcell.org/recur/).

For low-risk patients (e.g., stage I-II disease with no additional risk factors), we typically consider regular imaging every 6 months for the first 2-3 years, then every 9-12 months until the recurrence risk decreases below $2 \%$. For high-risk patients (e.g. stage III-IV disease and/or with prominent risk factors such as immunosuppression), we consider imaging every 3 months for the first $2-3$ years, and thereafter, every 6-12 months until recurrence risk decreases below $2 \%$.

$\mathrm{MCPyV}$ oncoprotein antibody testing can be used instead of or in conjunction with imaging studies. We typically perform the test every 3 months while the patient's risk remains or over $2 \%$.

For patients with local disease (stages I and II) who experience recurrences, 40-60\% patients develop local and/or nodal recurrences, while the remaining patients have distant metastasis. In advanced-stage disease (stage III), approximately $80 \%$ of recurrences are distant [68]. In a study of 215 patients with distant metastatic disease after initial diagnosis of locoregional disease (stage I-III), nonregional lymph nodes were the most common site (41\%), followed by skin/body wall (25\%) and liver (23\%). Lung and brain were relatively less often involved (7 and $5 \%$, respectively). This is an important difference from patterns of melanoma recurrence, in which approximately $10-30 \%$ of metastases occur in lung and brain [70].

\section{Representative cases of MCC management}

Case 1: typical management of low-risk local MCC, surveillance with MCPyV antibody serum testing An 81-year-old woman presented with a $2 \mathrm{~cm}$ local MCC involving her right upper eyelid. Her baseline scans did not show distant metastasis. She underwent treatment with surgical excision and reconstruction, as well as negative SLNB (0/5 lymph nodes from the right neck); American Joint Committee on Cancer, AJCC, 8th edition, pathologic stage I). She received adjuvant radiation therapy to the right upper eyelid given her approximately $20 \%$ risk of local recurrence [71]. MCPyV antibody serum test was performed 1 month after her initial diagnosis and was positive at a titer of 934 (Serum titer units [STU]). Three months later, her antibody titer had decreased to 213. Eight months after the WLE, her antibody titer had decreased further to the negative range $(<74)$. Her declining antibody titer corresponded to CT scans of the head, neck, chest, abdomen and pelvis with no evidence of recurrent or metastatic disease. She continued to receive surveillance via concurrent CT scans and MCPyV antibody serum testing every 6 months for the first 2 years. MCPyV antibody serum testing was used as the sole method for surveillance for an additional 3 years, sparing substantial expense and radiation exposure, and continued to be in the negative range.

Case 2: a high-risk patient who later developed metastatic disease, treated with anti-PD-1

A 61-year-old man presented with a $2 \mathrm{~cm}$ MCC of the left cheek. His baseline scans did not show distant metastasis, and MCPyV antibody titer within 3 months from his initial diagnosis was negative. He underwent WLE of the primary tumor, and SLNB that was positive for microscopic metastasis in one of two left preauricular lymph nodes (AJCC 8th edition, pathologic stage IIIA). Based on his disease stage and medical condition, his overall risk of recurrence was estimated to be approximately $50 \%$ over the next 5 years (https://merkelcell.org/recur/). He underwent postoperative radiation therapy to the primary site and draining lymph node basin (56 Gray). Three months after completion of adjuvant RT, routine surveillance imaging detected a new lesion in the liver. Biopsy confirmed metastatic MCC. Based on the potential durable benefit from PD-1/PD-L1 inhibitors over chemotherapy, the patient was offered pembrolizumab (anti-PD-1), to which he has had a CR for the last 18 months with excellent quality of life. 
Case 3: a high-risk, IS MCC patient whose disease was controlled with multiple lines of therapy An 81-year-old man with a history of indolent CLL was diagnosed with MCC of the skin of the right lower back $(0.7 \mathrm{~cm})$. He underwent WLE and SLNB (0/3 axillary and inguinal lymph nodes involved; AJCC 8th edition, pathologic stage I). Based on his disease stage and IS condition, his overall risk of recurrence was estimated to be approximately $40 \%$ over the next 5 years. Ten months after his initial diagnosis, he developed recurrent MCC of the right axillary and inguinal lymph node basins for which he initiated systemic therapy with pembrolizumab. He was treated with pembrolizumab and then avelumab for about 1 year with mixed response. His disease eventually progressed, and he underwent salvage therapy with lymph node dissection plus adjuvant RT. Despite treatment, his disease progressed to involve bones and multiple lymph node basins. Because he was ineligible for clinical trials due to CLL, he had an octreotide scan that confirmed somatostatin receptor expression and was treated with somatostatin analogue plus hypofractionated palliative RT [55]. His disease was controlled with minimal side effects from treatment for over 2 years after recurrence. Unfortunately, therapeutic options were eventually exhausted, and the patient succumbed to disease 3 years after the initial diagnosis.

\section{Conclusion \& future perspective}

While our model does not contradict principles outlined in current NCCN guidelines, it does clarify our clinical approach and decreases ambiguity that may arise at various decision points.

Despite recent progress in MCC management across specialties, unmet needs and further research opportunities exist in several areas including improving technology for tumor surveillance, decreasing toxicity of existing therapeutics, reducing incidence of disease recurrence, and advancing novel therapeutics.

Tumor surveillance: $50 \%$ of patients do not produce MCPyV antibodies and such patients have $42 \%$ higher risk of recurrence and thus would benefit from careful surveillance. Current surveillance modalities for seronegative patients rely on imaging studies and/or physical exam, and there is no established blood-based surveillance assay. The use of a highly sensitive circulating tumor DNA assay (recognizing patient-specific DNA mutations) may provide a useful surveillance approach for virus-negative MCC patients [72].

Decreasing morbidity of existing therapeutics and surgeries: Decreasing morbidity from surgery or radiation for large or locally advanced MCC may be achievable through increasing use of narrow-margin surgery plus adjuvant RT that may be delivered in a less-toxic manner such as via 8Gy SFRT. Decreasing morbidity from large surgeries may also be achieved using neoadjuvant systemic PD-1/PD-L1 inhibitors to shrink tumors prior to operation, which is an area of active investigation.

Prevention of disease recurrence: Using a therapeutic vaccine to decrease the risk of recurrence for virus positive MCC is being investigated by multiple groups. Clinical trials using PD-1/PD-L1 inhibitors for adjuvant treatment of stage III disease are also underway. Moreover, studies to identify optimal duration of immunotherapy in responders are needed to minimize post-treatment recurrence.

Novel treatments: Most importantly, there is a pressing need to develop treatment strategies to overcome PD-1/PD-L1-refractory MCC, which ultimately affects approximately 50\% of patients with metastatic/locally advanced MCC. Various combinations of immunotherapies and other modalities (such as RT, targeted therapy) with PD-1/PD-L1 inhibitors are being investigated in refractory patients.

Several forms of cellular therapy in which T-cells are reprogrammed specifically to recognized MCPyV positive tumor cells are also being explored.

\section{Author contributions}

SY Park and LC Zaba authored the primary draft of the manuscript, compiled and analyzed key information and data. C DoolittleAmieva, T Akaike and Y Moshiri authored part of the draft of manuscript. U Parvathaneni, S Bhatia, LC Zaba and P Nghiem provided senior oversight, helped compile the data and helped prepare the manuscript. All the authors have read and approved of the manuscript.

\section{Financial \& competing interests disclosure}

This research was made possible by funding from NIH/NCI R01CA162522, NIH/NCI P01CA225517, NIH/NCI Cancer Center Support Grant P30 CA015704, the MCC patient gift fund at University of Washington, and Kelsey Dickson Team Science Courage Research Team Award from the Prostate Cancer Foundation (award \#15CHAS04), and a 'Friends and Family of Nancy Haeseker' fund'. P Nghiem has received consulting fees from EMD Serono, Pfizer, Sanofi/Regeneron, 4SC and research grant support from Bristol-Meyers Squibb; S Bhatia has received consulting fees from Genentech, EMD Serono, Bristol Myers Squibb, Sanofi Genzyme 
and Exicure; and research funding to his institution (University of Washington) from Oncosec, EMD Serono, Merck, BMS, NantKwest, Immune Design, Novartis, Nektar and Exicure. The authors have no other relevant affiliations or financial involvement with any organization or entity with a financial interest in or financial conflict with the subject matter or materials discussed in the manuscript apart from those disclosed.

No writing assistance was utilized in the production of this manuscript.

Ethical conduct of research

IRB: all studies were performed in accordance with the Helsinki principles and were approved by the Institutional Review Board at the Fred Hutchinson Cancer Research Center (IRB \#6585). All the patients included in this study provided informed consent for their clinical data to be analyzed for research purposes.

Open access

This work is licensed under the Attribution-NonCommercial-NoDerivatives 4.0 Unported License. To view a copy of this license, visit http://creativecommons.org/licenses/by-nc-nd/4.0/

\section{Executive summary}

Background

- Merkel cell carcinoma (MCC) is an aggressive cancer associated with a high risk of local recurrence and distant metastases.

Role of a multidisciplinary team

- Best individualized care of MCC can be achieved by multidisciplinary management with close communication among doctors and patients.

Summary

- There has been significant progress in MCC care, including recommendations for initial staging work-up, local treatment with surgery and radiation, surveillance and systemic treatment options. Here, we provide updates on: the role of a multidisciplinary team, flow of MCC treatment, MCPyV oncoprotein antibodies as a tumor marker, significance of baseline imaging prior to surgery, wide local excision versus narrow excision, sentinel lymph node biopsy, treatment for draining lymph node basin or involved lymph nodes, potential role for hypofractionated RT, treatment strategy for locally unresectable and metastatic disease, immunosuppressed patients and surveillance with representative cases of MCC management.

\section{References}

Papers of special note have been highlighted as: • of interest; $\bullet \bullet$ of considerable interest

1. Harms KL, Healy MA, Nghiem P et al. Analysis of prognostic factors from 9387 Merkel cell carcinoma cases forms the basis for the new 8th Edition AJCC Staging System. Ann. Surg. Oncol. 23(11), 3564-3571 (2016).

2. Becker JC. Merkel cell carcinoma. Ann. Oncol. 21(Suppl. 7), vii81-vii85 (2010).

3. Heath M, Jaimes N, Lemos B et al. Clinical characteristics of Merkel cell carcinoma at diagnosis in 195 patients: the AEIOU features. J. Am. Acad. Dermatol. 58(3), 375-381 (2008).

4. Paulson KG, Park SY, Vandeven NA et al. Merkel cell carcinoma: current US incidence and projected increases based on changing demographics. J. Am. Acad. Dermatol. 78(3), 457-463, e452 (2018).

5. Becker JC, Stang A, Decaprio JA et al. Merkel cell carcinoma. Nat. Rev. Dis. Primers 3, 17077 (2017).

- A broad overview of Merkel cell carcinoma (MCC) biology and therapies.

6. Sundaresan P, Hruby G, Hamilton A et al. Definitive radiotherapy or chemoradiotherapy in the treatment of Merkel cell carcinoma. Clin. Oncol. (R. Coll. Radiol.) 24(9), e131-e136 (2012).

7. Paulson KG, Lewis CW, Redman MW et al. Viral oncoprotein antibodies as a marker for recurrence of Merkel cell carcinoma: a prospective validation study. Cancer 123(8), 1464-1474 (2016).

8. Samimi M, Molet L, Fleury M et al. Prognostic value of antibodies to Merkel cell polyomavirus T antigens and VP1 protein in patients with Merkel cell carcinoma. Br. J. Dermatol. 174(4), 813-822 (2016).

9. Lachance K, Akaike T, Cahill K et al. 590 Detecting Merkel cell carcinoma recurrence using a blood test: outcomes from 774 patients. J. Invest. Dermatol. 139(5), S101 (2019).

10. Moshiri AS, Doumani R, Yelistratova L et al. Polyomavirus-negative Merkel cell carcinoma: a more aggressive subtype based on analysis of 282 cases using multimodal tumor virus detection. J. Invest. Dermatol. 137(4), 819-827 (2017). 
11. NCCN. Merkel cell carcinoma (Version 1.2020). https://www.nccn.org/professionals/physician_gls/pdf/mcc.pdf

- Most updated and prominent MCC management guidelines that we endorse.

12. UOWDOLM. Anti Merkel cell panel. https://Testguide.Labmed.Uw.Edu/Public/View/Amerk

-• Logistical information for ordering Merkel cell polyomavirus oncoprotein antibody test in the USA.

13. CHRUDT. Manuel de Prélèvement. https://Www.Chu-Tours.Fr/Manuel-De-Prelevement/

-• Logistical information for ordering Merkel cell polyomavirus oncoprotein antibody test in Europe.

14. Yancovitz M, Finelt N, Warycha MA et al. Role of radiologic imaging at the time of initial diagnosis of stage T1b-T3b melanoma. Cancer 110(5), 1107-1114 (2007).

15. Mohr P, Eggermont AM, Hauschild A, Buzaid A. Staging of cutaneous melanoma. Ann. Oncol. 20(Suppl. 6), vi14-vi21 (2009).

16. George A, Girault S, Testard A et al. The impact of (18)F-FDG-PET/CT on Merkel cell carcinoma management: a retrospective study of 66 scans from a single institution. Nucl. Med. Commun. 35(3), 282-290 (2014).

17. Hawryluk EB, O'regan KN, Sheehy N et al. Positron emission tomography/computed tomography imaging in Merkel cell carcinoma: a study of 270 scans in 97 patients at the Dana-Farber/Brigham and Women's Cancer Center. J. Am. Acad. Dermatol. 68(4), 592-599 (2013).

18. Siva S, Byrne K, Seel M et al. 18F-FDG PET provides high-impact and powerful prognostic stratification in the staging of Merkel cell carcinoma: a 15-year institutional experience. J. Nucl. Med. 54(8), 1223-1229 (2013).

19. Poulsen M, Macfarlane D, Veness $M$ et al. Prospective analysis of the utility of 18-FDG PET in Merkel cell carcinoma of the skin: a Trans Tasman Radiation Oncology Group Study, TROG 09:03. J. Med. Imaging Radiat. Oncol. 62(3), 412-419 (2018).

20. Singh N, Alexander NA, Lachance K et al. Clinical benefit of baseline imaging in Merkel cell carcinoma: analysis of 584 patients. J. Am. Acad. Dermatol. S0190-9622(20), 32265-32269 (2020).

- Article demonstrating high rates of clinically occult MCC metastatic disease at the time of initial diagnosis.

21. Allen PJ, Bowne WB, Jaques DP, Brennan MF, Busam K, Coit DG. Merkel cell carcinoma: prognosis and treatment of patients from a single institution. J. Clin. Oncol. 23(10), 2300-2309 (2005).

22. Gillenwater AM, Hessel AC, Morrison WH et al. Merkel cell carcinoma of the head and neck: effect of surgical excision and radiation on recurrence and survival. Arch. Otolaryngol. Head Neck Surg. 127(2), 149-154 (2001).

23. Perez MC, De Pinho FR, Holstein A et al. Resection margins in Merkel cell carcinoma: is a 1-cm margin wide enough? Ann. Surg. Oncol. 25(11), 3334-3340 (2018).

24. Yiengpruksawan A, Coit DG, Thaler HT, Urmacher C, Knapper WK. Merkel cell carcinoma. Prognosis and management. Arch. Surg. 126(12), 1514-1519 (1991).

25. Lewis KG, Weinstock MA, Weaver AL, Otley CC. Adjuvant local irradiation for Merkel cell carcinoma. Arch. Dermatol. 142(6), 693-700 (2006).

26. Harrington C, Kwan W. Radiotherapy and conservative surgery in the locoregional management of Merkel cell carcinoma: the British Columbia Cancer Agency experience. Ann. Surg. Oncol. 23(2), 573-578 (2016).

27. Tarabadkar ES, Fu T, Lachance $\mathrm{K}$ et al. Narrow excision margins are appropriate for Merkel cell carcinoma when combined with adjuvant radiation: analysis of 188 cases of localized disease and proposed management algorithm. J. Am. Acad. Dermatol. S0190-9622(20), 32280-32285 (2020).

- Provides algorithm for local surgical margines \pm adjuvant radiation.

28. Conic RRZ, Ko J, Saridakis S et al. Sentinel lymph node biopsy in Merkel cell carcinoma: predictors of sentinel lymph node positivity and association with overall survival. J. Am. Acad. Dermatol. 81(2), 364-372 (2019).

29. Iyer JG, Storer BE, Paulson KG et al. Relationships among primary tumor size, number of involved nodes, and survival for 8044 cases of Merkel cell carcinoma. J. Am. Acad. Dermatol. 70(4), 637-643 (2014).

30. Ahmadzadehfar H, Hinz T, Wierzbicki A et al. Sensitivity and false negative rate of sentinel lymph node biopsy (SLNB) in malignant melanoma of different parts of the body. J. Nucl. Med. 55(1), 55 (2014).

31. Fang LC, Lemos B, Douglas J, Iyer J, Nghiem P. Radiation monotherapy as regional treatment for lymph node-positive Merkel cell carcinoma. Cancer 116(7), 1783-1790 (2010).

32. Leonard JH, Ramsay JR, Kearsley JH, Birrell GW. Radiation sensitivity of Merkel cell carcinoma cell lines. Int. J. Radiat. Oncol. Biol. Phys. 32(5), 1401-1407 (1995).

33. Cook MM, Schaub SK, Goff PH et al. Postoperative, single-fraction radiation therapy in Merkel cell carcinoma of the head and neck. Adv. Radiat. Oncol. 5(6), 1248-1254 (2020).

34. Iyer JG, Parvathaneni U, Gooley T et al. Single-fraction radiation therapy in patients with metastatic Merkel cell carcinoma. Cancer Med. 4(8), 1161-1170 (2015).

35. Rush Z, Fields RC, Lee N, Brownell I. Radiation therapy in the management of Merkel cell carcinoma: current perspectives. Expert Rev. Dermatol. 6(4), 395-404 (2011). 
36. Nghiem PT, Bhatia S, Lipson EJ et al. PD-1 blockade with pembrolizumab in advanced Merkel cell carcinoma. N. Engl. J. Med. 374(26), 2542-2552 (2016).

37. Kaufman HL, Russell J, Hamid O et al. Avelumab in patients with chemotherapy-refractory metastatic Merkel cell carcinoma: a multicentre, single-group, open-label, Phase 2 trial. Lancet Oncol. 17(10), 1374-1385 (2016).

38. D'angelo SP, Russell J, Lebbé C et al. Efficacy and safety of first-line avelumab treatment in patients with stage IV metastatic Merkel cell carcinoma: a preplanned interim analysis of a clinical trial. JAMA Oncol. 4(9), e180077 (2018).

39. Topalian SL, Bhatia S, Hollebecque A et al. Abstract CT074: non-comparative, open-label, multiple cohort, Phase I/II study to evaluate nivolumab (NIVO) in patients with virus-associated tumors (CheckMate 358): efficacy and safety in Merkel cell carcinoma (MCC). Cancer Res. 77(Suppl. 13), CT074-CT074 (2017).

40. Nghiem P, Bhatia S, Lipson EJ et al. Durable tumor regression and overall survival in patients with advanced Merkel cell carcinoma receiving pembrolizumab as first-line therapy. J. Clin. Oncol. 37(9), 693-702 (2019).

- Most mature data regarding PD-1 inhibitors in advanced MCC which reports increased overall survival in patients receiving pembrolizumab.

41. Iyer JG, Blom A, Doumani R et al. Response rates and durability of chemotherapy among 62 patients with metastatic Merkel cell carcinoma. Cancer Med. 5(9), 2294-2301 (2016).

42. Topalian SL, Bhatia S, Amin A et al. Neoadjuvant nivolumab for patients with resectable Merkel cell carcinoma in the CheckMate 358 Trial. J. Clin. Oncol. 38(22), 2476-2487 (2020).

43. Cowey CL, Mahnke L, Espirito J, Helwig C, Oksen D, Bharmal M. Real-world treatment outcomes in patients with metastatic Merkel cell carcinoma treated with chemotherapy in the USA. Future Oncol. 13(19), 1699-1710 (2017).

44. Xu MJ, Wu S, Daud AI, Yu SS, Yom SS. In-field and abscopal response after short-course radiation therapy in patients with metastatic Merkel cell carcinoma progressing on PD-1 checkpoint blockade: a case series. J. Immunother. Cancer 6(1), 43 (2018).

45. Lopiccolo J, Schollenberger MD, Dakhil S et al. Rescue therapy for patients with anti-PD-1-refractory Merkel cell carcinoma: a multicenter, retrospective case series. J. Immunother. Cancer 7(1), 170 (2019).

46. Bhatia S, Miller NJ, Lu H et al. Intratumoral G100, a TLR4 agonist, induces antitumor immune responses and tumor regression in patients with Merkel cell carcinoma. Clin. Cancer Res. 25(4), 1185-1195 (2019).

47. Bhatia S, Longino NV, Miller NJ et al. Intratumoral delivery of plasmid IL12 via electroporation leads to regression of injected and noninjected tumors in Merkel cell carcinoma. Clin. Cancer Res. 26(3), 598 (2020).

48. Nguyen MHK, Leong SP, Abendroth R, Kashani-Sabet M, Kim KB. Complete clinical response to intralesional talimogene laherparepvec injection in a patient with recurrent, regionally advanced Merkel cell carcinoma. JAAD Case Rep. 5(10), 849-851 (2019).

49. Westbrook BC, Norwood TG, Terry NLJ, Mckee SB, Conry RM. Talimogene laherparepvec induces durable response of regionally advanced Merkel cell carcinoma in 4 consecutive patients. JAAD Case Rep. 5(9), 782-786 (2019).

50. Lara KM, In GK, Matcuk GR Jr, Mehta A, Hu JS. Talimogene laherparepvec in combination with pembrolizumab leads to a complete response in a patient with refractory Merkel cell carcinoma. JAAD Case Rep. 4(10), 1004-1006 (2018).

51. Blackmon JT, Dhawan R, Viator TM, Terry NL, Conry RM. Talimogene laherparepvec for regionally advanced Merkel cell carcinoma: a report of 2 cases. JAAD Case Rep. 3(3), 185-189 (2017).

52. Knackstedt R, Sussman TA, Mccahon L, Song JM, Funchain P, Gastman B. Pre-treated anti-PD-1 refractory Merkel cell carcinoma successfully treated with the combination of PD-1/PD-L1 axis inhibitors and TVEC: a report of two cases. Ann. Oncol. 30(8), 1399-1400 (2019).

53. Tarabadkar ES, Thomas H, Blom A et al. Clinical benefit from tyrosine kinase inhibitors in metastatic Merkel cell carcinoma: a case series of 5 patients. Am. J. Case Rep. 19, 505-511 (2018).

54. Nathan PD, Gaunt P, Wheatley K et al. UKMCC-01: a Phase II study of pazopanib (PAZ) in metastatic Merkel cell carcinoma. J. Clin. Oncol. 34(Suppl. 15), 9542-9542 (2016).

55. Akaike T, Qazi J, Anderson A et al. High somatostatin receptor expression and efficacy of somatostatin analogues in patients with metastatic Merkel cell carcinoma. Br. J. Dermatol. 184(2), 319-327 (2020).

56. Salavati A, Prasad V, Schneider CP, Herbst R, Baum RP. Peptide receptor radionuclide therapy of Merkel cell carcinoma using (177)lutetium-labeled somatostatin analogs in combination with radiosensitizing chemotherapy: a potential novel treatment based on molecular pathology. Ann. Nucl. Med. 26(4), 365-369 (2012).

57. Basu S, Ranade R. Favorable response of metastatic merkel cell carcinoma to targeted 177Lu-DOTATATE therapy: will PRRT evolve to become an important approach in receptor-positive cases? J. Nucl. Med. Technol. 44(2), 85-87 (2016).

58. Cook M, Baker K, Redman M et al. Differential outcomes among immunosuppressed patients with merkel cell carcinoma: impact of immunosuppression type on cancer-specific and overall survival. Am. J. Clin. Oncol. 42(1), 82-88 (2018).

59. Engels EA, Frisch M, Goedert JJ, Biggar RJ, Miller RW. Merkel cell carcinoma and HIV infection. Lancet 359(9305), 497-498 (2002).

60. Garrett GL, Blanc PD, Boscardin J et al. Incidence of and risk factors for skin cancer in organ transplant recipients in the United States. JAMA Dermatol. 153(3), 296-303 (2017). 
61. Paulson KG, Iyer JG, Blom A et al. Systemic immune suppression predicts diminished Merkel cell carcinoma-specific survival independent of stage. J. Invest. Dermatol. 133(3), 642-646 (2013).

62. Muirhead R, Ritchie DM. Partial regression of Merkel cell carcinoma in response to withdrawal of azathioprine in an immunosuppression-induced case of metastatic Merkel cell carcinoma. Clin. Oncol. (R. Coll. Radiol.) 19(1), 96 (2007).

63. Singh JA, Saag KG, Bridges SL Jr et al. 2015 American College of Rheumatology guideline for the treatment of rheumatoid arthritis. Arthritis Care Res. 68(1), 1-25 (2016).

64. Elandt K, Aletaha D. Treating rheumatic patients with a malignancy. Arthritis Res. Ther. 13(3), 223 (2011).

65. Wang JL, Yin WJ, Zhou LY et al. Risk of non-melanoma skin cancer for rheumatoid arthritis patients receiving TNF antagonist: a systematic review and meta-analysis. Clin. Rheumatol. 39(3), 769-778 (2020).

66. Zawacki L, Lachance K, Akaike T, Nghiem P. 497 Efficacy of immunotherapy in Merkel cell carcinoma patients with chronic immunosuppression. J. Invest. Dermatol. 140(7), S67 (2020).

67. Menzies AM, Johnson DB, Ramanujam S et al. Anti-PD-1 therapy in patients with advanced melanoma and preexisting autoimmune disorders or major toxicity with ipilimumab. Ann. Oncol. 28(2), 368-376 (2017).

68. Lachance KS, Thomas HJ, Mcevoy AM, Hippe DS, Nghiem P. 594 An integrated approach to predict and detect Merkel cell carcinoma recurrences. J. Invest. Dermatol. 138(5), S101 (2018).

69. Bichakjian CK, Lowe L, Lao CD et al. Merkel cell carcinoma: critical review with guidelines for multidisciplinary management. Cancer 110(1), 1-12 (2007).

70. Lewis CW, Qazi J, Hippe DS et al. Patterns of distant metastases in 215 Merkel cell carcinoma patients: implications for prognosis and surveillance. Cancer Med. 9(4), 1374-1382 (2020).

71. Takagishi SR, Marx TE, Lewis C et al. Postoperative radiation therapy is associated with a reduced risk of local recurrence among low risk Merkel cell carcinomas of the head and neck. Adv. Radiat. Oncol. 1(4), 244-251 (2016).

72. Riethdorf S, Hildebrandt L, Heinzerling $\mathrm{L}$ et al. Detection and characterization of circulating tumor cells in patients with Merkel cell carcinoma. Clin. Chem. 65(3), 462-472 (2019). 